\title{
Initial Research of np Scattering with Polarized Deuterium Target at ANKE/COSY
}

\author{
B. Gou \\ for the ANKE Collaboration \\ Institute of Modern Physics, Chinese Academy of Sciences, Lanzhou, 730000, China \\ School of Nuclear Science and Technology, Lanzhou University, Lanzhou, 730000, China \\ gouboxing@impcas.ac.cn
}

Published 29 February 2016

\begin{abstract}
The quasi-free np charge-exchange reaction $p \vec{d} \rightarrow\{p p\}_{s} n$ has to be employed to extend the investigations of np scattering at ANKE to the highest energy available at COSY. As the proof-of-principle experiment, the initial research has been conducted at proton energy $T_{p}=600 \mathrm{MeV}$ using a polarized Deuterium target. The vector and tensor analyzing powers $A_{y}$ and $A_{y y}$ were measured for momentum transfers $q \geqslant 160 \mathrm{MeV} / \mathrm{c}$. These data connect smoothly with the previous measurements at $q \leqslant 140 \mathrm{MeV} / \mathrm{c}$ performed using a polarized deuteron beam. The reported data are well reproduced by the impulse approximation using the SAID np amplitudes. The results therefore proves that it is possible to continue the np programme at higher energies at ANKE.
\end{abstract}

Keywords: Quasi-free; charge-exchange; proton-neutron scattering; polarized deuterium target; analyzing power.

\section{Introduction}

Thorough understanding of the nuclear forces is essential to both nuclear and hadronic physics. Nucleon-nucleon (NN) scattering is a powerful tool to study the nuclear forces. The ANKE collaboration has embarked on a comprehensive programme ${ }^{2}$ on NN scattering for many years, aiming at investigating the amplitudes of both proton-proton ( $\mathrm{pp}$ ) and neutron-proton (np) scattering. This paper is intended to report the latest results of the np study at the ANKE magnetic spectrometer. ${ }^{1}$ Due to the lack of free neutron source, the np study is usually performed by using substituents such as $\mathrm{D} /{ }^{3} \mathrm{He}$, which provide the quasi-free neutrons. It was pointed out $^{3}$ that the free np spin-dependent amplitudes can be deduced from the observables of the charge-exchange deuteron breakup reaction $d p \rightarrow\{p p\} n$ by phase-shift analysis (PSA), provided that the final proton pair is in ${ }^{1} S_{0}$ state. ${ }^{\text {a }}$ The transition

This is an Open Access article published by World Scientific Publishing Company. It is distributed under the terms of the Creative Commons Attribution 3.0 (CC-BY) License. Further distribution of this work is permitted, provided the original work is properly cited.

${ }^{\text {a }}$ Proton pair at state ${ }^{1} S_{0}$ is denoted by $\{p p\}_{S}$ in this paper. 


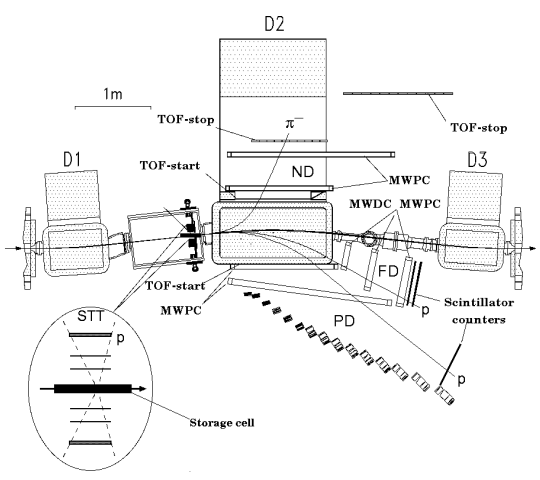

Fig. 1. Schematic plot of ANKE.

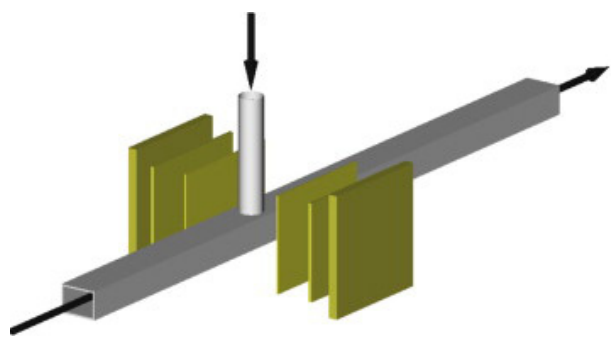

Fig. 2. 3D plot of the storage cell and STT.

from the initial deuteron states ${ }^{3} S_{1},{ }^{3} D_{1}$ to the final proton pair state ${ }^{1} S_{0}$ involves a spin flip, therefor it is sensitive to the spin-dependent amplitudes. Using this method, the ANKE collaboration has conducted a series of experiments using the polarized deuteron beams provided by the COoler SYnchrotron (COSY) ${ }^{4}$ and uppolarized/polarized hydrogen targets at ANKE up to the nucleon energy $T_{N}=1.135$ $\mathrm{GeV}$, which corresponds to the most energetic deuteron beam at COSY. Valuable information has been obtained out of these experiments. ${ }^{5-8}$ The success encourages the ANKE Collaboration to continue the study at high energies. The proton beam and deuterium target have to be employed in order to extend the np programme at ANKE up to the highest energy available at $\operatorname{COSY}\left(T_{N}=2.8 \mathrm{GeV}\right)$. To validate the feasibility, the initial research has been done using an unpolarized proton beam at $600 \mathrm{MeV}$ and a polarized deuterium target at ANKE. Fig. 1 shows the schematic plot of ANKE, the charge-exchange reaction $p \vec{d} \rightarrow\{p p\}_{s} n$ as well as the polarimetry reaction $p \vec{d} \rightarrow p d$ are registered in a pair of silicon tracking telescopes (STT), ${ }^{9}$ placed on left and right sides of an aluminium storage cell of $25 \mu \mathrm{m}$ thick, which is used for increasing the target density. Fig. 2 illustrates the placement of the STT and the storage cell. The forward (FD) and positive (PD) sub-detectors are exploit to detect the reaction $p \vec{d} \rightarrow d \pi^{+} n$, which can also be used for the polarimetry.

\section{Targets and Polarimetry}

The polarized deuterium target is provided by the polarized internal target (PIT),${ }^{10-11}$ which was also employed to prepare the polarized hydrogen target in the first stage of the np programme at ANKE. ${ }^{8}$ The PIT can be configured to produce polarized deuterium target of different modes $\left(Q_{y}, Q_{y y}\right) .{ }^{\mathrm{b}}$ In this experiment four polarization modes $(+1,+1),(-1,+1),(0,-2)$ and $(0,+1)$ were used. During the experiment the first tow modes $(+1,+1)$ and $(-1,+1)$ were tuned to have identical target densities and were switched between each other every 10 seconds in order

${ }^{\mathrm{b}} Q_{y}$ and $Q_{y y}$ are the vector and tensor polarizations respectively. 
to minimize the systematic error, the same is true for the third and fourth modes $(0,-2)$ and $(0,+1)$. Data were also taken with an unpolarized deuterium target for comparison with the polarized data. Besides the deuterium targets, a nitrogen target was used to simulate the background caused by the interaction between the beam halo and the storage cell.

Both the vector $\left(A_{y}\right)$ and the tensor $\left(A_{x x}\right.$ and $\left.A_{y y}\right)$ analyzing powers of the pd-elastic reaction $p \vec{d} \rightarrow p d$ within the acceptance of the STT have been measured by Argonne, ${ }^{12}$ SATURNE $^{13-14}$ and $\mathrm{ANKE}^{6}$ at neighboring energies to $T_{N}=600$ $\mathrm{MeV}$. Fig. 3 shows the measured analyzing powers $A_{y}, A_{x x}$ and $A_{y y}$ fitted by polynomials as functions of the deuteron scattering angle in the center-of-mass frame. The particles detected by the STTs are identified via the energy deposit in different silicon layers, Fig. 4 shows the good separation between proton and deuteron. The pd-elastic reaction is selected using the missing-mass method from the events with one deuteron detected by the STT. For the reactions with two final particles induced by unpolarized proton and polarized deuteron with vector $\left(Q_{y}\right)$ and tensor $\left(Q_{y y}\right)$ polarizations, such as $p \vec{d} \rightarrow p d$ and $p \vec{d} \rightarrow\{p p\}_{s} n$ where the proton pair is considered as a system without internal freedom, the differential cross section is given by ${ }^{15}$

$$
\begin{aligned}
\frac{d \sigma^{P}}{d \Omega}(\theta, \phi)={\frac{d \sigma^{0}}{d \Omega}}^{P}(\theta)\{1+ & \frac{3}{2} Q_{y} A_{y}(\theta) \cos \phi \\
& \left.+\frac{1}{4} Q_{y y}\left[A_{x x}(\theta)(1-\cos 2 \phi)+A_{y y}(\theta)(1+\cos 2 \phi)\right]\right\}
\end{aligned}
$$

where $\theta$ and $\phi$ are the polar and azimuthal angle in the center-of-mass system respectively, and $\frac{d \sigma}{d \Omega}(\theta)$ is the unpolarized differential cross section. For convenience, we denote the the polarizarion modes in the same pair which switch between each other by a and b. The differences and averages of the polarizations $\Delta Q_{y}=Q_{y}^{a}-Q_{y}^{b}$, $\Delta Q_{y y}=Q_{y y}^{a}-Q_{y y}^{b}$ and $\left\langle Q_{y}\right\rangle=\frac{1}{2}\left(Q_{y}^{a}+Q_{y}^{b}\right),\left\langle Q_{y y}\right\rangle=\frac{1}{2}\left(Q_{y y}^{a}+Q_{y y}^{b}\right)$ can be obtained via fitting the ratio of the difference to the sum of the counts for $a$ and $b$

$$
R=\frac{N_{a}-N_{b}}{N_{a}+N_{b}}
$$

measured using both the left and right STTs by

$r(\theta, \phi)=\frac{\frac{3}{2} A_{y}(\theta) \cos \phi \Delta Q_{y}+\frac{1}{4}\left[A_{x x}(\theta)(1-\cos 2 \phi)+A_{y y}(\theta)(1+\cos 2 \phi)\right] \Delta Q_{y y}}{2+3 A_{y}(\theta) \cos \phi\left\langle Q_{y}\right\rangle+\frac{1}{2}\left[A_{x x}(\theta)(1-\cos 2 \phi)+A_{y y}(\theta)(1+\cos 2 \phi)\right]\left\langle Q_{y y}\right\rangle}$

Since the polarization modes a and b were alternating every 10 seconds and their densities are identical, the beam condition as well as the detector performance were regarded the same for them, therefore the only systematic uncertainty arises from the deuteron analyzing powers $A_{y}(\theta), A_{x x}(\theta)$ and $A_{y y}(\theta)$, which are plugged into the fitting function Eq. 3 as the polynomials in Fig. 3. The systemic uncertainties were estimated by varying the parameters of these polynomials within their standard deviations and repeating the fitting procedure. Table 1 presents the measured 
Table 1. The ideal and the measured polarizarions in the form of difference and average, as well as the statistical and systematic uncertainties.

\begin{tabular}{|c|r|r|c|c|c|c|}
\hline Pol. & \multicolumn{3}{|c|}{ Modes 1,2 } & \multicolumn{3}{c|}{ Modes 3,4 } \\
\cline { 2 - 6 } & Ideal & Measured & Sys. err. & Ideal & Measured & Sys. err. \\
\hline$\Delta Q_{y}$ & +2 & $1.46 \pm 0.01$ & 0.03 & 0 & $-0.07 \pm 0.01$ & 0.01 \\
$\left\langle Q_{y}\right\rangle$ & 0 & $-0.03 \pm 0.01$ & 0.01 & 0 & $-0.02 \pm 0.02$ & 0.01 \\
$\Delta Q_{y y}$ & 0 & $0.17 \pm 0.02$ & 0.01 & -3 & $-1.68 \pm 0.02$ & 0.14 \\
$\left\langle Q_{y y}\right\rangle$ & +1 & $0.88 \pm 0.03$ & 0.11 & -0.5 & $-0.13 \pm 0.06$ & 0.03 \\
\hline
\end{tabular}

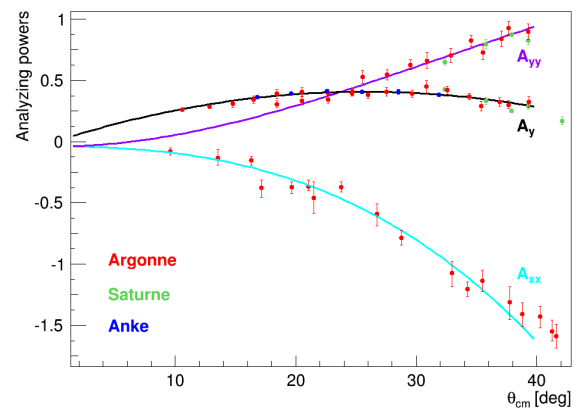

Fig. 3. Analyzing powers of $p \vec{d} \rightarrow p d$.

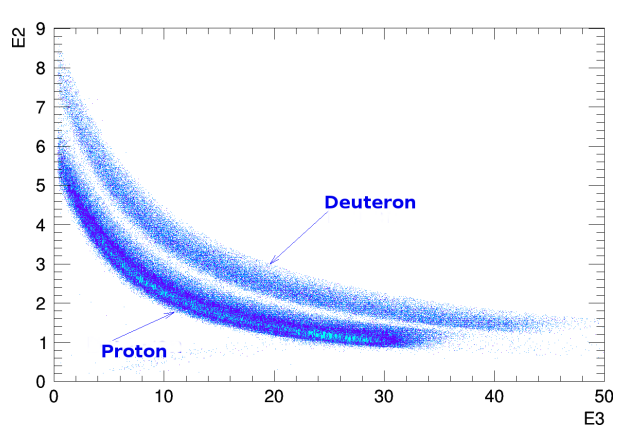

Fig. 4. Particle identification by STT.

polarizations in the form of the differences and mean values. The ideal values and the uncertainties are given as well.

The vector polarizations of the modes 1 and 2 were measured via the quasi-free reaction $p \vec{p} \rightarrow d \pi^{+}$to be $\left|Q_{y}\right|=0.7489 \pm 0.0668$, which is consistent with results obtained using the pd-elastic reaction.

\section{Analyzing Power Measurement for Charge-exchange Reaction}

Fig. 5 demonstrates the event selection for the charge-exchange reaction using the missing-mass method from the events with two protons in the STTs and the background subtraction using the nitrogen data. Fig. 6 shows the three-momentum transfer $q$ from the incident proton to the target neutron in the np charge-exchange process versus the excitation energy $E_{p p}$ of the final proton pair. Theoretical calculation $^{3}$ predicted that the deuteron vector analyzing power $A_{y}^{d}$ should be zero if the diproton is at ${ }^{1} S_{0}$ state, which is insured experimentally by requiring $E_{p p}<3 \mathrm{MeV}$. The difference of the ratio $\frac{N_{1}-N_{2}}{N_{1}+N_{2}}$ between the left and right STT is evaluated to measure $A_{y}^{d}$, small residual effects arising from the tensor analyzing powers are corrected using impulse approximation predictions. The measurement result shown in Fig. 7 agrees well with the theoretical calculation.

Restrict the azimuthal angel close to 0 and $\pi$, which correspond to the centers of the left and right STTs respectively, and take into account the conclusion $A_{y}^{d}=0$, 


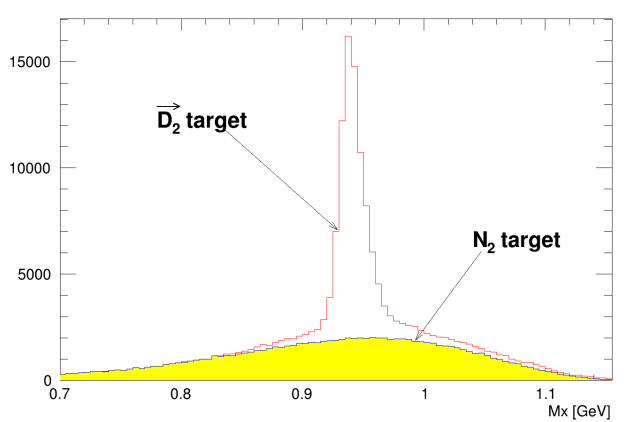

Fig. 5. Missing mass of $p \vec{d} \rightarrow\{p p\}_{s} X$, the background from the storage cell is simulated using the scaled nitrogen data, the scaling factor is determined by the fitting beyong the peak.

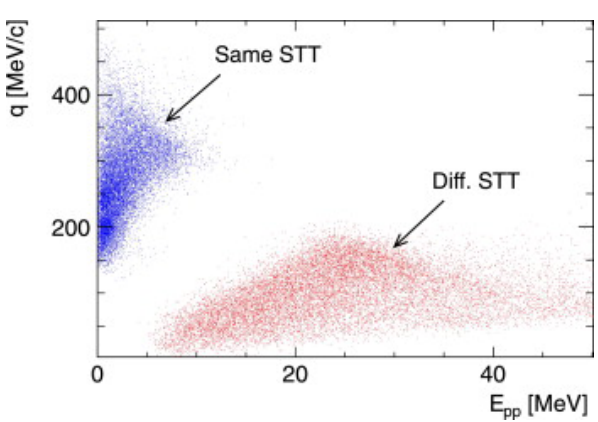

Fig. 6. The momentum transfer q versus the pp excitation energy $E_{p p}$ for the $p \vec{d} \rightarrow\{p p\}_{s} X$ events with two protons in the same STT (blue) and the different STTs (red).

the ratio defined by Eq. 2 is expressed analytically as

$$
r(\theta)=\frac{\frac{1}{2} A_{y y}(\theta) \Delta Q_{y y}}{2+A_{y y}(\theta)\left\langle Q_{y y}\right\rangle}
$$

Using the measured polarization difference $\Delta Q_{y y}$ and average $\left\langle Q_{y y}\right\rangle$ the tensor analyzing power $\left(A_{y y}\right)$ of the charge-exchange reaction can be extracted by comparing Eq. 4 and the ratio $R$. Since the polarization pair $(3,4)$ yields large sensitivity to the tensor signal, only this pair is used for Ayy determination. The values of $A_{y y}$ obtained from the left and right STT data agree with each other within the errors, the averaged results are presented in Fig. 8.

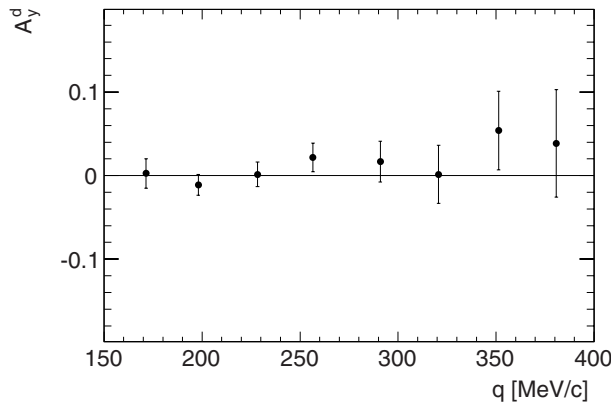

Fig. 7. Deuteron vector analyzing power $A_{y}^{d}$ of $p \vec{d} \rightarrow\{p p\}_{s} n$ at energy per nucleon $T_{N}=$ $600 \mathrm{MeV}$ as a function of the momentrum transfer q. The results agrees with the theoretical calculation, ${ }^{3}$ which predicted that the deuteron vector analyzing power $A_{y}^{d}$ should be zero if the final diproton is at ${ }^{1} S_{0}$ state.

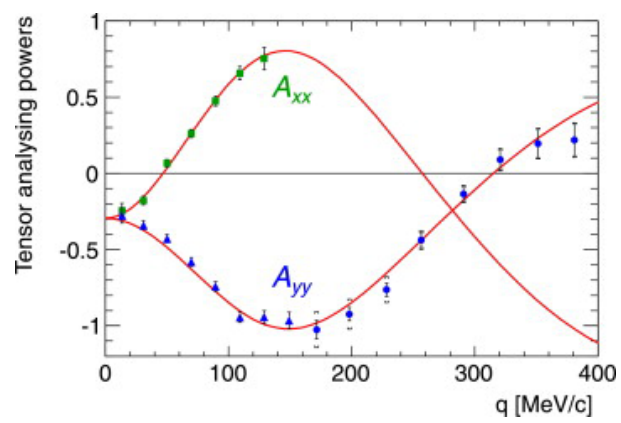

Fig. 8. Deuteron tensor analyzing powers $A_{x x}^{d}$ and $A_{y y}^{d}$ of $p \vec{d} \rightarrow\{p p\}_{s} n$. The squres and the triangles are the results from the previous measurements using deuteron beams while the filled circles are the results using a deuteron target. The curves are the theorecical calculations by the impulse approximation. 


\section{Summary and Outlook}

The charge-exchange dueteron breakup reaction $p \vec{d} \rightarrow\{p p\}_{s} n$ has been studied at $T_{p}=600 \mathrm{MeV}$ using a polarized deuterium target at ANKE. The newly measured analyzing power $A_{y y}$ at the momentum transfers $q \geqslant 160 \mathrm{MeV} / \mathrm{c}$ joint smoothly with the results obtained at the same energy per nucleon in inverse kinematics, using a polarized deuterium beam.. Furthermore, the measurements agree well with the impulse approximation calculations based on the SAID SP07 solution ${ }^{16-18}$ for the $n p$ scattering amplitudes. This research proves that it is possible to extend the np programme at ANKE to higher energies, where the np amplitudes are less known.

\section{Acknowledgments}

The author is grateful to other members of the ANKE Collaboration for their help with the experiment and to the COSY crew for providing excellent working conditions. This work has been supported by the CSC programme (No. 2011491103).

\section{References}

1. S. Barsov et al., Nucl. Instr. Methods A 462364 (2001).

2. A. Kacharava et al., Spin Physics from COSY to FAIR, COSY proposal 152 (2005).

3. D. V. Bugg and C. Wilkin, Nucl. Phys. A 467575 (1987).

4. R. Maier, Nucl. Instr. Methods A 3901 (1997).

5. D. Chiladze et al., Phys. Lett. B 637170 (2006).

6. D. Chiladze, et al., Phys. Rev. Spec. Top., Accel. Beams 9050101 (2006).

7. D. Chiladze et al., Eur. Phys. J. A 4023 (2009).

8. D. Mchedlishvili et al., Eur. Phys. J. A 4949 (2013).

9. R. Schleichert et al., IEEE Trans. Nucl. Sci. 50301 (2003).

10. M. Mikirtychyants et al., Nucl. Instrum. Methods A 72183 (2013).

11. R. Engels et al., Rev. Sc. Instr. 744607 (2003).

12. M. Haji-Said, et al., Phys. Rev. C 362010 (1987).

13. J. Arvieux, et al., Nucl. Phys. A 431613 (1984).

14. J. Arvieux, et al., Nucl. Instrum. Methods A 27348 (1988).

15. G.G. Ohlsen, Rep. Prog. Phys. 35717 (1972).

16. R.A. Arndt, et al., Phys. Rev. C 62034005 (2000).

17. R.A. Arndt, et al., Phys. Rev. C 76025209 (2007).

18. SAID data base, http://gwdac.phys.gwu.edu. 